\title{
Reducing Insulin Administration Errors in Inpatients from a Nursing Perspective: A Literature Review
}

\author{
Jieli LI ${ }^{1 *}$ and Rasika Jayasekara ${ }^{2}$ \\ ${ }^{1}$ Master of Nursing Practice Candidate, Monash University, Australia \\ ${ }^{2}$ Department of Nursing \& Midwifery, University of South Australia, Australia \\ *Corresponding author: Jieli LI, Master of Nursing Practice Candidate, Monash University, Australia. \\ To Cite This Article: Jieli LI.Reducing Insulin Administration Errors in Inpatients from a Nursing Perspective: A Literature Review. Am J Biomed Sci \\ \& Res. 2019 - 6(2). AJBSR.MS.ID.001016. DOI: 10.34297/AJBSR.2019.06.001016.
}

Received: 阱 October 03, 2019; Published: 眥 November 14, 2019

\section{Introduction}

It is widely agreed that medication error can not only cause financial, psychological and emotional stress to patients, healthcare professionals, and healthcare providers, but also has the potential to cause severe physical injury and possible death to patients [1]. Over the past century there has been a dramatic increase in researching how to prevent medication error in various healthcare settings, among which the topic of medication administration error remains as the most active area [2,3]. A large and growing body of literature has investigated the root causes of medication administration errors and proposed strategies of reducing these errors accordingly [2-4]. However, giving the fact that insulin medication errors occurred more often than any other type or class of drug [5,6], and can cause detrimental or even irreversible damages to patients [7], so far, there has been little discussion about how to reduce insulin administration errors in inpatients from a nursing perspective. The purpose of this paper is to examine the prevalence, consequences, and contributing factors of insulin administration errors world widely from a nursing perspective. It also describes the high burden of diabetes mellitus on Australians and the increased use of insulin in Melbourne and Sydney public hospitals. Last but not least, this paper continues the most effort of the author to understand the known evidence-based strategies of preventing insulin administration error in inpatients.

The Prevalence, Consequences, and Contributing Factors of Insulin Administration Errors

Data from the Australian Institute of Health and Welfare Diabetes indicates approximately 1.2 million accounting for $6 \%$ of Australian adults have been diagnosed with diabetes in 2017-2018 [8]. Over 1 million hospitalisations (principal and/ or additional diagnosis) were associated with diabetes in 20162017 [8]. Inpatients with diabetes are generally older, heavier, and more vulnerable with significant comorbid conditions [9]. An Australian survey including 11 hospitals, 2308 adult inpatients in metropolitan Melbourne reported 25\% (range: 15.7-35.1\%) of diabetes prevalence, and almost half of them were treated with insulin alone or in combination with oral hypoglycaemic agents [9]. Compare to oral diabetic agents which are often contraindicated during hospitalisation when patients are fasting, having renal insufficiency and being exposed to investigations involving radiocontrast dye, insulin has superiority in forms of acting mechanism, controllability, effectiveness, and limited side effects [10]. Therefore, it has been prescribed frequently in managing hyperglycaemia in the hospital setting [10]. However, previous research has shown that insulin medication errors occurred more often than any other type or class of drug [5,6]. Evidence from the National Patient Safety Agency in the United Kingdom confirmed that a total of 16,600 incidents involving insulin were identified in England and Wales from November 2003 to November 2009, among which 18 incidents with fatal and devastating outcomes and 1,042 with moderate harm [11]. The more recent United Kingdom National Diabetes Inpatient Audit in 2016 revealed that $22.7 \%$ of inpatient medication charts had at least one insulin medication error [12]. In comparison, the rate of insulin-related medication error in $\mathrm{St}$ Vincent's Hospital Sydney Australia has been reported even higher with $41.5 \%$ of insulin charts revealed one or more prescription or administration errors in 2013, 28.6\% in 2014 and 35.4\% in 2016 [10].

Insulin, in Australia, has been classified by the Australian Commission on Safety and Quality in Health Care as one of the high- 
alert APINCH medications that "to be associated with high potential medication-related harm" [13]. The most common consequences of insulin medication errors include unnecessary blood glucose fluctuation, hypoglycaemia, hyperglycaemia or even death [5]. A small amount of insulin overdose has been found to cause diaphoresis, tachycardia, anxiety and hunger $[7,14]$. With a larger amount of insulin overdose, the patient can experience more serious nervous system symptoms and progress to confusion, lethargy and delirium to seizures and coma [7,14]. A lethal dose of insulin or prolonged hypoglycaemia can lead to irreversible brain damage and death $[7,14]$. Numerous medication incidents involving insulin have been reported by studies with different levels of impacts on patients [7,14-16]. A case of insulin dosing error in a patient with severe hyperkalaemia triggered a rapid response team code (RRT code) resulting in prolonged hospitalisation for the patient [7].

Another incident case study reported that due to insulin overdose patient developed bradycardia, hypotension, and remained in coma for 90 hours [16]. Because of insulin's high risk of causing harm to patients, the Australian National Safety and Quality Health Service (NSQHS) Standard on Medication Safety requires all health providers to take appropriate actions to ensure safe storing, prescribing, dispensing, and administering of insulin [17]. However, it has been pointed out by the Institute for Safe Medication Practices [18] that some hospitals have no safety nets in place at all regarding on how to administer high-alert medications including insulin, and they purely rely on staff vigilance to keep patients safe. The evidence presented from a preceptor training programme study strongly supports the idea that there is an inconsistency of medication administration practice, and interpretation of medication administration policies, procedures, and protocols in hospitals [19].

In the literature, insulin administration errors have been categorised into different types. Qualitative analysis from the National Patient Safety Agency in the UK indicated the top three insulin administration errors were the wrong dose, omitted or delayed insulin [11]. Insulin administration error has also been associated with different contributing factors, of which a significant portion was due to product confusion [11]. Cousins [11] suggested that the wrong insulin product was given due in large part to the "look-alike" and "sound-alike" accounting for $60 \%$ of incidents reported. Table 1 below gives a more detailed summary of the most common known contributing factors leading to insulin error [11].

\begin{tabular}{|c|c|c|}
\hline Wrong dose & Omitted insulin/ Delayed insulin & Product confusion \\
\hline $\begin{array}{l}\text { Inconsistent abbreviation of "units" to be "U" or } \\
\text { "IU" }\end{array}$ & $\begin{array}{l}\text { Neglect Supplemental Insulin Orders and Stat/ } \\
\text { Phone Orders }\end{array}$ & Novorapid VS Novomix \\
\hline $\begin{array}{l}\text { Illegible handwriting " } U \text { " and "IU" to be read as } \\
\text { "0" or " } 10 \text { " leading to unintended } 10 \text { times and } \\
100 \text { times overdose }\end{array}$ & $\begin{array}{l}\text { Nurses signed medication chart before giving } \\
\text { insulin and omitted the administration }\end{array}$ & Humalog VS Humalog Mix \\
\hline $\begin{array}{l}\text { Poor/lack of documentation of given insulin } \\
\text { dose }\end{array}$ & $\begin{array}{l}\text { Insulin administration omitted when patient "nil } \\
\text { by mouth" }\end{array}$ & Humulin S, I and M3 \\
\hline Duplicate dose administration & Failure in communication & Humalog VS Humulin \\
\hline Calculation error for insulin IV infusion & Slips and memory lapses & Glulisine VS Glargine \\
\hline Incorrect programming of an infusion pump & & Lantus VS Lente \\
\hline Transcription errors & & Hypurine - neutral, isophane, lente \\
\hline
\end{tabular}

Adding to the above, new advanced technology has made insulin to be given at a much easier and more convenient way but also brings in the extra risk of making medication error. The nursing staff has been reported making mistakes due to lack of knowledge of the insulin pen devices and the unawareness of safe administration of medication pen devices [20]. A patient was given 46 units of insulin instead of 6 units because of the unfamiliarity of the NovoLOG FlexPen [15]. It was later discovered that the number to the right of the dosing window was mistakenly interpreted as the dialing dosing while the actual dosing window was neglected [15]. Similarly, the Clinical Excellence Commission in New South Wales has reported incidents where staff administered insulin using standard pen needles without removing the inner cover, mistaking them for safety pen needles, resulting in patients received inadequate insulin and ended up with hyperglycaemia and other complications [20]. In summary, as a high-risk medication, insulin can cause different levels of damages to patients and needs to be safely regulated. While medication errors involving insulin are still happening frequently in different forms owing to different contributing factors. Effective risk-reduction strategies need to be implemented to prevent and minimise the occurrence of insulin error.

\section{Evidence-Based Risk-Reduction Strategies}

The ISMP suggests that medication risk-reduction strategies should impact as many steps of the medication-use process as feasible to eliminate the underlying causes [18]. Medication administration is usually the last step of the medication process but numerous factors can lead to an insulin administration error [19]. The previous sections have listed the known contributing factors of insulin administration errors such as transcribing errors, drug calculation mistakes, non-adherence to protocols, and failure in 
communication. While it is argued that the majority of medication administration incidents are preventable if a certain amount of risk-reduction strategies are implemented [5]. Strategies such as enhancing clinical governance, providing staff education, promoting inter-professional communication, and creating a safe hospital environment have been proven to be effective [5]. To understand these strategies in a more specific way, the following section presents a comprehensive review of the current medication error reduction strategies with the focus on preventing insulin errors. The first one is nursing workflow modification.

It is well understood that nursing workflow is impacted by factors such as the number of medications available/prescribed for each patient, the frequency of order amendments, the technology being used (electronic systems) in the workplace, and the policies and procedures [19]. On top of these, additional tasks involved in medication administration process overwhelmingly burden the nursing workflow [19]. For example, tasks such as patient assessment, medication retrieval, five rights verification, documentation, and monitoring side effects are putting extra time pressure on nurses [19]. Therefore, ways of error elimination related to nursing workflow can be avoiding excessive workload, anticipating patient needs to inform supply needs, timely restocking medications, providing easily accessible drug information, and the use of advanced technology [19]. A safe insulin administration guidance published by the UK National Patient Safety Agency introduces the use of barcode scanning technology to increase safe identification and the electronic medical record to accelerate communication, decrease nursing workload [11]. It is also anticipated that handwritten medication charts which cause ambiguity and extra time consumption will eventually be replaced by high acuity electronic recording devices [11]. A computerised system with features such as medication incompatibility alert, medication maximum dose alert, medication acting mechanism references has been found enabling nurses to work more efficiently with fewer medication errors [21].

Secondly, enhance interdisciplinary teamworkand coordination. Cohesive interdisciplinary teamwork and coordination are crucial in maintaining medication safety [22]. Failure in communication and teamwork has been identified by numbers of studies in root cause analysis as the major issues leading to fatal medication errors [22]. Quantitative and qualitative evidence from a systematic review shows that communication failure (including written communication) accounts for over $50 \%$ of all causes associated with medication errors [4]. Another cross-sectional study of emergency department medication error concluded that the leading causes of these errors were not following procedure/protocol $(17 \%)$ and poor (verbal) communication (11\%) [23]. The most common communication-related factors that have been identified in a retrospective clinical audit in Australian paediatric hospitals were bedside communication problems, clinical handover problems and medical record documentation problems [24]. Therefore, it is recommended that nurses to be involved in multidisciplinary patient safety rounds and to have a place in multidisciplinary medication committees [19]. By doing this, Nurses can be informed timely about medication changes with rationale and they can communicate directly with the team about any concern [19]. When nurses are held in medication committees, getting involved in medication incident analyses, risk management of medication, medication safety improvement, they tend to be more informative, vigilant and compliant to protocols [19].

Thirdly, ensure consistency in prescribing, calculating, abbreviating and measurements. The unsafe abbreviation that appeared in prescription or medical charts can cause ambiguity and misinterpretation of medication names, dosage or units [11]. Under the NSQHS Medication Safety Standards Action 4.15 Medication management processes, safe prescribing is required to be implemented in workplace especially relating to high-risk medicines [17]. That is to say, a standardised or specialised chart for insulin prescription need to be available, dose-calculating tools and units are to be consistent, and protocols or standard set are to be utilised to prevent insulin administration error [17]. This is consistent with the recommended key safety strategies for safeguarding high-alert medications from ISMP: employ evidence-based, standardised order sets, concentrations, units, and measurements [18]. The observational study by Harada [5] has testified that the introduction of a standardised sliding scale insulin order sheet can significantly reduce the incidence of insulin errors (12/165 [7.3\%] vs 4/159 [2.1\%], $\mathrm{P}=.048)$.

Fourthly, reinforce the adherence to policies and protocols. The Australian Commission on Safety and Quality in Health Care (2019) has produced nation subcutaneous insulin chart to support the effective management of blood glucose levels (BGL) and appropriate administration of insulin in hospitalised patients [13]. The User guide to the national subcutaneous insulin chart: Acute facilities in conjunction with the User guide to the national subcutaneous insulin chart: Sub-acute hospitals and mental health facilities have specified how to prevent Insulin administration errors in inpatients [17]. This include doing a thorough check-up for routine insulin orders, supplemental insulin orders, and stat/ phone orders before administering any insulin as patients are commonly prescribed more than one type/one dose of insulin at a time [17] It is also recommended to calculate the total insulin dose (routine \pm supplemental \pm stat/phone) and follow hospital procedures to determine whether different types of insulin can be mixed in the same syringe [17].

Adding to that, insulin as an injectable medication needs to be double-checked by two trained nurses and seven rights need to be verified before administering insulin as per most Australian hospitals' protocols [17]. However, the reality is, regardless of 
how thorough the policies and protocols have been designed, and how well the nurses understand the relevant guidelines, without adherence medication safety will be compromised. According to Harris [19], nurses' adherence to protocols is actually problematic with only $57 \%$ of nurses verified patients' information using wristbands before administering medication. A large number of nurses justified themselves with reasons such as "I know my patients well enough", or "it is a common practice of my work colleagues" [19]. However, data from the National Patient Safety Agency indicates that around 10\%-15\% medication error occurred due to mismatching patients and medications [19] It is obvious that "knowing the patients" does not prevent nurses from giving the "right drugs" to the wrong patient or the wrong drugs to the "right patient". Addressing to the above, evidenced-based strategies surrounding nonadherence to medication protocols include strengthening the leadership roles in cultivating a safety climate, mandating educational programmes, recognising and reporting medication errors [19]. ISMP [18] encourages nurses to adopt a safe practice habit of self-briefing prior to medication administration as it is an effective way to reinforce memory cues and knowledge about patient conditions and medications. The UK National Patient Safety Agency suggests the enrolment of an e-learning package for nursing staffs to reinforce their intention of protocol adherence [11].

Lastly, Strategies specifically related to insulin pen devices also have been widely addressed owing to the frequency of using insulin pens and the special form of insulin packaging. Following the Safe Administration of Medication Pen Devices guidelines that, where possible, patients should administer their own medication pen devices using a standard pen needle or safety pen needle [20]. If it is necessary for staff to administer a pen device, a safety pen needle should be used for each dose [20]. The implication for nursing staff to prevent insulin administration error is making sure the removal of both the inner and outer covers when giving an insulin injection with a pen. This is supported by Cohen \& Smetzer [25] who suggested developing staff orientation and continuing education programs regarding the safe use of insulin pen devices. The National Patient Safety Agency in the UK has carried out numerous studies to develop information handbook and programmes to better inform patients, carers and healthcare professionals about the insulin products, insulin pen devices to reduce error in practice [11].

The Alfred Health in Melbourne Australia is a good illustration of conducting their own research in developing programmes to reduce insulin error. An observational study conducted in the Alfred Health Australia has reported being successful in reducing potentially fatal errors associated with high doses of insulin [26]. This new structure validation programme detected and rectified 8 instances where high doses of insulin were prescribed in error [26]. It is therefore concluded that consideration should be given about adopting this structure verification process in any setting where insulin is prescribed and administered [26]. Other strategies that have been mentioned in literature with beneficial outcomes include the introduction of design of safe labelling and packaging of insulin [11]. In addition, research also emphasises the benefit of collaborating with pharmaceutical manufacturers and regulators to promote design that will improve the safe use of insulin in practice [11]. Although these strategies are not explicitly considered from a nursing perspective they can significantly prevent nurses from making accidental insulin administration errors [11].

\section{Conclusion}

In summary, it has been shown from this review that diabetes is highly prevalent in the general Australian population and significant numbers of hospitalisations in Australia were associated with diabetes. Owing to the benefits of insulin treatment, inpatients are very likely to be treated with insulin alone or in combination with oral hypoglycaemic agents. However, insulin administration error has been reported to be alarmingly frequent and caused detrimental damage or even death to patients. The consequences of insulin error range from suffering mild symptoms such as diaphoresis, tachycardia to more severe symptoms such as delirium to seizures and coma, and to the worst outcomes of irreversible brain damage and death. In Australia, insulin has been classified by the Australian Commission on Safety and Quality in Health Care as one of the high-alert APINCH medications where specific guidelines are to be followed to ensure safe store, prescribing, dispensing, and administering. However, the evidence presented from literature has shown there is an inconsistency of insulin administration practice and some hospitals have no safe nets or risk-reduction strategies in place at all and they purely rely on staff vigilance to keep patients safe.

In terms of the forms of insulin administration error, the most common error types were the wrong dose, omitted or delayed insulin. The current common known contributing factors leading to insulin error were transcribing errors, drug calculation mistakes, non-adherence to protocols, and failure in communication. While it is argued that insulin administration incidents are largely preventable if a certain amount of risk-reduction strategies are implemented. This paper clusters the current medication error reduction strategies with the focus on preventing insulin errors in five categories based on a comprehensive review of the most recent literature. They arenursing workflow modification; interdisciplinary teamwork and coordination enhancement; standardise prescribing, calculating, abbreviating and measurements; adherence to policies and protocols reinforcement; and other insulin specific programmes and guidelines developments. This review will serve as a base for our understanding of the importance of safe insulin administration. It further indicates that considerably more effort will be needed in all healthcare settings to implement effective evidence-based riskreduction strategies to eliminate insulin administration error. 


\section{References}

1. Rutledge DN, Retrosi T, Ostrowski G (2018) Barriers to medication error reporting among hospital nurses. J Clin Nurs 27(9-10): 1941-1949.

2. Alomari A, Wilson V, Davidson P, Lewis J (2015) Families, nurses and organisations contributing factors to medication administration error in paediatrics: a literature review. International Practice Development Journal 5(1): 1-15.

3. Baraki Z, Tsegay L, Gerensea H, Kebede A, Teklay H (2018) Medication administration error and contributing factors among paediatric inpatient in public hospitals of Tigray, northern Ethiopia. BMC Paediatr 18(1): 321

4. Keers RN, Williams SD, Cooke J, Ashcroft DM (2013) Causes of medication administration errors in hospitals: A systematic review of quantitative and qualitative evidence. Drug Saf 36(11): 1045-1067.

5. Harada S, Suzuki A, Nishida S, Kobayashi R, Tamai S, et al. (2017) Reduction of medication errors related to sliding scale insulin by the introduction of a standardized order sheet. Journal of Evaluation in Clinical Practice 23(3): 582-585.

6. Garrouste Orgeas M, Philippart F, Bruel C, Max A, Lau N, et al. (2012) Overview of medical errors and adverse events. Ann Intensive Care 2(1):

7. Hewitt D, Barnard C, Bilimoria K (2017) Insulin dosing error in a patient with severe hyperkalaemia. JAMA 318(24): 2485-2486.

8. (2019) Diabetes. Australian Institute of Health and Welfare.

9. Bach L, Ekinci E, Engler D, Gilfillan C, Hamblin P, et al. (2014) The high burden of inpatient diabetes mellitus: The Melbourne public hospitals diabetes inpatient audit. Medical Journal of Australia 201(6): 334-338.

10. Taylor J, Campbell L, Zhang L, Greenfield J (2018) High diabetes prevalence and insulin medication errors in hospital patients. Intern Med J 48(12): 1529-1532.

11. Cousins D, Rosario C, Scarpello J (2011) Insulin, hospitals and harm: A review of patient safety incidents reported to the National Patient Safety Agency. Clin Med (Lond) 11(1): 28-30.

12. (2016) National Diabetes Inpatient Audit (NaDIA) - 2016. Digital.

13. (2019) National subcutaneous insulin charts. Australian Commission on Safety and Quality in Health Care.

14. Lu M, Inboriboon PC (2011) Lantus insulin overdose: A case report Journal of Emergency Medicine 41(4): 374-377.
15. Cohen MR (2012) Medication errors: Insulin pen. Nursing, 42(12), 12 12.

16. Gundgurthi A, Kharb S, Dutta MK, Pakhetra R, Garg MK (2012) Insulin poisoning with suicidal intent. Indian J Endocrinol Metab 16(7): 120122.

17. (2019) The NSQHS standards, Medication safety standard, Medication management processes. Australian Commission on Safety and Quality in Health Care.

18. (2013) Your high-alert medication list - relatively useless without associated risk-reduction strategies. Institute for Safe Medication Practices (ISMP).

19. Harris R (2014) Improving preceptors' knowledge on medication error reduction strategies. MedSurg Nursing 23(6): 402-407.

20. (2019) Safe Administration of Medication Pen Devices. Clinical Excellence Commission.

21. Narayanan J, Dobrin S, Choi J, Rubin S, Pham A, et al. (2017) Structured clinical documentation in the electronic medical record to improve quality and to support practice-based research in epilepsy. Epilepsia 58(1): 68-76.

22. Adhikari R, Tocher J, Smith P, Corcoran J, Macarthur J (2014) A multi-disciplinary approach to medication safety and the implication for nursing education and practice. Nurse Educ Today 34(2): 185-190.

23. Pham JC, Story JL, Hicks RW, Shore AD, Morlock LL, et al. (2011) National Study on the frequency, types, causes, and consequences of voluntarily reported emergency department medication errors. J Emerg Med 40(5): 485-492.

24. Manias E, Cranswick N, Newall F, Rosenfeld E, Weiner C, et al. (2019) Medication error trends and effects of person-related, environment-related and communication-related factors on medication errors in a paediatric hospital. J Paediatr Child Health 55(3): 320-326.

25. Cohen M, Smetzer J (2012) Error prevention strategies for strong iodine solution; Do not use an insulin pen for multiple patients. Hospital Pharmacy 47(4): 260-263.

26. Dooley M, Wiseman M, Mcrae A, Murray D, Van De Vreede M, et al. (2011). Reducing potentially fatal errors associated with high doses of insulin: A successful multifaceted multidisciplinary prevention strategy. BMJ Qual Saf 20(7): 637-644. 